CYP and parents were consulted about their experiences of oral mucositis, and potential future treatment options, through NIHR-funded patient involvement activities, and low-level laser therapy (LLLT) was identified as an approach warranting further exploration.

LLLT is a NICE-recommended treatment option for oral mucositis, based mainly on research in adults. It is not currently used for CYP in England or Wales. We determined to conduct an updated systematic review evaluating the evidence for the efficacy and tolerance/safety of LLLT in CYP undergoing treatment for cancer.

Methods Standard systematic review methods were used (protocol registration: PROSPERO CRD42018099772). Two reviewers systematically searched medical databases, contacted authors and reviewed study references. Studies had to include patients with a diagnosis of cancer; use LLLT prophylactically or as a treatment; and, for assessing efficacy, include CYP less than 18 years old, and be a randomised control trial. For the safety/tolerability analysis, all study types and ages were included.

Results 3390 results for initial screening were identified, with 115 full texts assessed for efficacy, and 159 for safety. LLLT is generally well tolerated, and may be effective at treating oral mucositis, but there is uncertainty around the optimal protocol due to the heterogeneity of results. Different protocols were used, adopting various frequencies, dose, timings and wavelengths of LLLT.

Conclusion CYP must not be left behind when new evidence and treatment options become available for adults.

LLLT has many ways of administration, and further research is needed to assess the efficacy and appropriate use in CYP.

Acknowledgements We are grateful to NIHR Research Design Service for Yorkshire and the Humber for a grant allowing patient and public involvement.

\section{G179(P) EMERGENCY MANAGEMENT OF ACUTE PAINFUL CRISIS IN PAEDIATRIC SICKLE CELL PATIENTS; INVESTIGATING ANALGESIC TREATMENT DELAYS AT A DISTRICT GENERAL HOSPITAL}

E Tsouana, K Ominu-Evbota, N Chaudhary, R Clubb, N Tuffin. Department of Paediatrics, Basildon Hospital, Basildon, UK

\subsection{6/archdischild-2020-rcpch.150}

Aims To evaluate the initial management of sickle cell (SC) patients presenting to our Paediatric Assessment Unit (PAU) with vaso-occlusive crisis (VOC), with focus on time to first dose of analgesia.

Methods Retrospective review of data extracted from electronic medical records for VOC cases presenting to our PAU between July 2016 to July 2019. Multi-linear logistic regression analysis was performed to investigate the effect of various parameters on times to analgesia.

Results A total of 95 VOC cases from 52 unique patients were analyzed. Demographics and clinical characteristics are demonstrated in table 1 . Time to analgesia was over the 30 minute standard in $63 \%$ of cases. The mean time from arrival to administration of analgesia was 54 minutes and from prescription to administration was 11 minutes. For the majority of patients $(89.5 \%)$, initial analgesia included a fast-acting opiate (intranasal diamorphine or fentanyl lozenges). Receiving

\begin{tabular}{ll} 
Abstract G179(P) Table 1 & \\
\hline Age & \\
Range & $2-16$ years \\
Mean & 9.6 years \\
\hline Gender & \\
Female (\%) & $50(53 \%)$ \\
Genotype & \\
HbSS & $85(89.5 \%)$ \\
HbSC & $5(5.3 \%)$ \\
HbS/beta Thalassaemia & $5(5.3 \%)$ \\
Mode of arrival & \\
Car & $77(81.1 \%)$ \\
Ambulance & $18(18.9 \%)$ \\
Time of presentation & \\
In hours (9am-5pm) & $37(38.9 \%)$ \\
Out of hours (5pm-9am) & $58(61.1 \%)$ \\
Pain score on presentation & \\
Mild (0-3) & $2(2.1 \%)$ \\
Moderate (4-6) & $24(25.3 \%)$ \\
Severe (7-10) & $69(72.6 \%)$ \\
\hline
\end{tabular}

analgesia within 30 minutes of arrival was significantly associated with high pain score on presentation $(p=0.06)$. There was no significant association with mode of arrival $(p=0.47)$, time of presentation $(p=0.7)$ or the use of an integrated care pathway tool $(p=0.13)$. Although age overall did not have a significant effect $(p=0.36)$, subgroup analysis showed that the 12-16 years age group was more likely to receive analgesia late $(\mathrm{p}=0.04)$.

Conclusions Door-to-analgesia times were often prolonged for paediatric SC patients presenting with VOC. High pain score on arrival was linked with shorter times to analgesia, but most other parameters examined did not significantly affect times to analgesia. Targeted quality improvement projects for timely emergency pain management in paediatric SC patients are needed, with particular emphasis on the 12-16 years age group, as it appears to be particularly susceptible to analgesic treatment delays.

\section{G180(P) EXPLORING EQUITY, PATIENT EXPERIENCE AND ENGAGEMENT IN PAEDIATRIC SICKLE CELL DISEASE (SCD): A QUALITY IMPROVEMENT PROJECT}

${ }^{1} \mathrm{~L}$ Huckerby, ${ }^{1} \mathrm{H}$ Lynch, ${ }^{1} \mathrm{R}$ Clubb, ${ }^{1} \mathrm{G}$ Haddock, ${ }^{1} \mathrm{~A}$ Harper, ${ }^{2} \mathrm{~K}$ Turner, ${ }^{3} \mathrm{~B}$ Basnett, ${ }^{1,4} \mathrm{~A}$ Leigh. ${ }^{1}$ Paediatrics, Barts Health NHS Trust, London, UK; ${ }^{2}$ Strategy, Barts Health NHS Trust, London, UK; ${ }^{3}$ Public Health, Barts Health NHS Trust, London, UK; ${ }^{4}$ Paediatrics, University College Hospital, London, UK

\subsection{6/archdischild-2020-rcpch.151}

Aims

- To evaluate paediatric SCD patient experiences of outpatient, inpatient and emergency services at a district general hospital

- To understand the factors contributing to a $20 \%$ rate of missed clinic appointments for SCD

- To implement changes to paediatric SCD services that improve outpatient clinic attendance and patient experiences

Methods Primary data were collected from June 2018 to August 2019, through the following:

- Semi-structured questionnaire with patients and carers 